\title{
Performance Analysis of Different Spatial Filters used in Speckle Denoising
}

\author{
Shilpa Joshi \\ Research Scholar, \\ Department of Electronics \& Communication \\ Engineering, VESIT, Chembur,Mumbai, \\ Maharashtra,India
}

\author{
R. K. Kulkarni, PhD \\ Professor, \\ Department of Electronics \& Communication \\ Engineering, VESIT, Chembur, Mumbai, \\ Maharashtra,India
}

\begin{abstract}
Medical images are often deteriorated by noise due to various sources of interferences and other phenomena that affect the measurement processes. The varieties include Speckle noise, Gaussian noise, Salt and pepper noise. It is a difficult task to separate noise from an image while maintaining the desired information and quality of an image. In the field of biomedical imaging, the ultrasound (US) B-Scan images are used for tissue characterization. These images are obtained with a simple linear or sector scan US probe, which show a granular appearance called speckle. Speckle is modeled as a signal dependent noise, which tends to reduce the image resolution and contrast, thereby reducing the diagnostic values of the US imaging modality. Over a period, various speckle reduction techniques have been developed by researchers did not represent a comprehensive method that takes all the constraints into consideration. The results obtained are presented in the form of filtered images, statistical tables and diagrams. Based on the statistical measures and visual quality of the US B-scan images the Wiener filter performed well over the other filter techniques.
\end{abstract}

The paper represents comparison of spatial filters approaches i.e. filtering approach using linear and Non-linear filters accounting Peak Signal to Noise Ratio, Root Mean Square Error, and Universal Quality Index, Structural Similarity Index and Run Time as performance parameters. This paper proves that Weiner filtering method is very effective for all types of noise.

\section{General Terms}

Medical Image Processing, Image Modalities, Noise, Image Enhancement.

\section{Keywords}

Speckle noise, Ultrasound image, spatial filtering, speckle suppression.

\section{INTRODUCTION}

Application of ultrasound(US) in medicine began as early as in second world war and rapidly developed ever since[1] and became essential technique in medical diagnosis[4] Ultrasound imaging, as a tool for medical diagnosis, is widely used in clinical practice, and in some situations it has become a standard procedure. Although diagnostic ultrasound is considered a harmless technique and permits real-time and

Noninvasive anatomical scanning, B-mode images are pervaded by the speckle artifact, which results from destructive interference effects between returning echoes. This artifact introduces fine-false structures whose apparent resolution is beyond the capabilities of the imaging system, reducing image contrast and masking the real boundaries of the tissue under investigation. Its occurrence may substantially compromise the diagnostic effectiveness, introducing a great level subjectivity in the interpretation of the images. Speckle can be defined as a destructive interference artifact and its severity depends on the relative phase between two overlapping returning echoes [5].

Speckle reduction techniques can be applied to ultrasound images in order to reduce the noise level and improve the visual quality for better diagnoses. Several methods have been proposed for speckle reduction. Some chose to enhance the ultrasound image using statistical models for both noise and signal. Some other methods use an adaptive technique [8] and others use a statistical approach to treat multiplicative noise. But the approaches used in this paper are based on spatial domain filtering. There are two basic approaches to speckle reduction in US B-Scan images, one is spatial filtering method and the other one is transform-domain based filtering method. The usual way of removing the speckle noise from US image using the spatial filter technique works well only if the underlying signal is smooth. In this paper, we have analyzed the performance of spatial mean filters, median filters as well as some special filters.

The remaining part of the paper is organized as follows: In section II, explanation of the methods and materials that are used in the comparative approach is given. In section III, the experimental results and discussion are given. In section IV, the conclusions are given.

\section{NOISE FILTERING TECHNIQUES IN SPATIAL DOMAIN \\ 2.1 Image Noise}

The Noise is any degradation in the image signal, caused by external disturbances. Image noise is a random variation of brightness or color information in images that are not present in the object imaged. It can be produced by the sensor and circuitry of a scanner or digital camera. Image noise is generally of the following types [7]: Gaussian noise, Salt-andpepper noise, Short noise, Uniform noise, Film grain noise and speckle. noise which is type of multiplicative noise. Today, US medical imaging is a common method for diagnosis over the other imaging modalities like Positron emission tomography (PET), Magnetic Resonance imaging (MRI) and Computed tomography (CT) due to its low cost and availability. In monochromatic radiations like US and laser, the radiations scattered from a surface with a roughness of the order of a wavelength produce interference patterns called speckle. 


\subsection{Speckle Noise}

Speckle noise is a granular noise. This noise generally degrades Synthetic Aperture Radar (SAR) images to large extent. This noise is generally caused due to random ups and downs in the signal coming back from an object that is smaller than a single image-processing element. It is also caused by consistent processing of backscattered signals from a no of distributed targets. This noise also increases the mean grey level of affecting image. This noise creates a lot of difficulty in interpreting the image. [20]. To understand the speckle noise properties and its despeckling techniques biomedical researchers have been developing mathematical models. In this study, general model for speckle noise given by A. K. Jain [14] has been adopted as such and is given in equation 1 .

$$
(x, y)=f(x, y) n m(x, y)+n a(x, y) \quad 1
$$

Where $f(x, y)$ is the noise free image to be recovered, $S(x, y)$ is the noisy image, $\boldsymbol{n m}(\mathrm{x}, \mathrm{y})$ and $\boldsymbol{n a}(\mathrm{x}, \mathrm{y})$ are multiplicative and additive noises respectively. For any speckle, the contrast ratio $(\sigma)$ is defined as

\section{$\sigma=$ standard deviation of $I /$ mean value of $I 2$}

Where I is the intensity of the field

\section{FILTERING ALGORITHMS}

Spatial filters provide a convenient way to remove the random noises from the image intensity profile. In this paper, for speckle denoising four non-linear spatial mean filters as well as four linear median filters along with some special filters are studied. So far each having its own advantages and limitation. One should choose the technique according to the type and amount of noise present in the image. One should also consider the other factors like performance in denoising the image, computational time, and computational cost. Denoising can be done in various domains like Spatial Domain, Frequency Domain and Wavelet Domain. The Spatial domain method is discussed below. Filtering is a technique in image processing which is used for different tasks like noise reduction, interpolation, and re- sampling. It is mostly used in all image processing systems. The choice of filter depends upon the type and amount of noise present in an image because different filters can remove different types of noise efficiently. Spatial Domain has following types of filters.

\subsubsection{Linear Filters:}

Linear filters are used to remove certain type of noise. Here filtering is generally done by blurring the image. These filters blur the edges and destroy the fine details

\subsubsection{Gaussian Filter:}

Gaussian filter is a non-uniform low pass filter. Gaussian filter is used to blur images and remove noise and detail. It does not remove salt $\&$ pepper noise effectively [3].

\subsubsection{Average Filter:}

The output of average filter is simply the average of pixels contained in the neighborhood of filter mask. It calculates the average of all intensities of the neighborhood of the central pixel and replaces the pixel with that average value. It is mostly used in removing irrelevant details from an image. It has a limitation that it blurs the edges of the image [19].

\subsubsection{Non-Linear Filters:}

In recent years, a variety of nonlinear filters such as median filter, min filter, max filter have been developed to overcome the shortcoming of linear filter. Non-linear filters exhibit better performance than linear filters [10]. They are discussed below:

\subsubsection{Mean Filter:}

It is one of the simplest filter among the existing spatial filters. It uses a filter window which is usually square. The filter window replaces the center value in the window with the average mean of all the pixels values in the kernel.

\subsubsection{Median Filter:}

It is also known as order statistics filter. It is most popular and commonly used nonlinear filter. It removes noise by smoothing the images. This filter also lowers the intensity variation between one and other pixels of an image. In this filter, the pixel value of image is replaced with the median value The median value is calculated by first arranging all the pixel values in ascending order and then replace the pixel being calculated with the middle pixel value.

\subsubsection{Adaptive Filters:}

These filters work accordingly the statistical characteristics of image inside the filter region defined by the $m \times n$ rectangular window. They are more complex and gives better performance than existing spatial filters. The most commonly used spatial filter is adaptive median filter which is discussed below.

\subsubsection{Adaptive Median Filter:}

It performs well on images containing high density salt \& pepper noise. It preserves the details of an image while smoothing non impulse noise. It changes its windows size during its operation depending on the certain conditions [19]. It works in two stages. First it calculates the minimum, maximum and median values of sub image window of the corrupted image.

\section{SELECTION OF FILTERING ALGORITHM}

Selection of the de-noising algorithm depends on the application. The principle approach of image de-noising is filtering. According to need of detailing the performance of various spatial enhancement approaches are analyzed and discussed. The measurement of image enhancement is difficult to measure. There is no common algorithm for the enhancement of the image. According to cause of introduction of noise Speckle noise becomes a dominating factor in degrading the image visual quality and perception in many other images. Noise is introduced at all stages of image acquisition [3]. The denoising algorithm can be chosen according to input image characteristics and content. In image processing, image is corrupted by different type of noises. An appropriate method for speckle reduction is one which enhances the signal to noise ratio while conserving the edges and lines in the image. The sample mean and variance of a single pixel are equal to the mean and variance of the local area that is centered on that pixel. A vast literature has emerged recently on signal denoising using nonlinear techniques, in the setting of speckle noise. The image analysis process can be broken into three primary stages which are preprocessing, data reduction, and features analysis. Removal of noise from an image is the one of the important tasks in image processing. Depending on nature of the noise, such as additive or multiplicative noise, there are several approaches for removal of noise from an image [5]. The main objective of Image denoising techniques is necessary to remove such noises while retaining as much as possible the important 
signal features The statistical measurement could be used to measure enhancement of the image. The Mean Square Error (MSE) and Peak Signal-to-Noise Ratio (PSNR) are used to evaluate the enhancement performance. The noise level is measured by the standard deviation of the image: Existing filters are not much efficient in removing speckle noise, they may cause to loss of details in low contrast border regions. Irrespective of the method used to reduce speckle noise from images, the ideal speckle reduction method must preserve radiometric information and the textural information i.e. the edges between different area and spatial signal variability the spatial filters are of two types which are adaptive or nonadaptive. A non-adaptive filter takes the parameters of the whole image signal into consideration and leave out the local properties of the sensors [6]. These kinds of recommend filters are not appropriate for not-stationary scene signal. According to organ to be scaned Performance of all filtering techniques is tested with ultrasound image regard to organ. The performance of noise removing filtering techniques is measured using quantitative performance measures such as PSNR and MSE as well as in term of visual quality of the images. Many of the methods fail to remove speckle noise present in the ultrasound medical image, since the information about the variance of the noise may not be identified by the methods. The Ultrasound image as an input which contains speckle noise. To remove speckle noise we can apply NLMean filter and Lee filter on the given image to remove speckle noise. From the above said procedure we can get three different images as output images then we consider MSE, RMSE, SNR and PSNR parameters to compare the quality of output images. The adaptive filters accommodate changes in local properties as well as the nature of sensors. Adaptive filters reduce speckle while preserving the edges and these filters modify the image based on statistical extracted from the local environment of each pixel [4]. A filter that adapts the stretch to the region of interest produces a better enhancement. These filters are Mean, Median, Lee, Leesigma, Frost and Gamma. V: Parametric Description The performance parameters are most important criteria to justify results through evaluation. The parameters considered here are peak signal to noise ratio (PSNR) and mean square error (MSE) by which the objective quality of the reconstructed image is measured. The Universal Quality Index (UQI) and structural similarity index (SSIM) are mainly used for measuring the similarity between two images. The difference with respect to other techniques mentioned previously such as MSE or PSNR is that these approaches estimate absolute errors; on the other hand, SSIM is a perception-based model that considers image degradation as perceived change in structural information, Structural information is the idea that the pixels have strong inter-dependencies especially when they are spatially close. These dependencies carry important information about the structure of the objects in the visual scene. The qualitative performance of these selected filters are evaluated based on level of noise density are given in table No.1 and 2 respectively for medium and high noise density. For the quantitative comparison of the selected filters is reprsentated in graphical format. Figure 2, 3, 4 represents the performance analysis in graphical form. The Quantitative performance of the spatial filters is evaluated through Peak signal to noise ratio (PSNR). It can be defined by following eq.

$$
\begin{aligned}
& P S N R=10 \log 10\left(255^{2} / M S\right) \\
& M S E=\Sigma i \Sigma j(r i j)-(x i j) M * N /
\end{aligned}
$$

\section{$S S I M=(2 \mu x \mu+c 1)(2 \sigma x y+c 2)(\mu x+2 \mu x+2 c 1)(\sigma x+2$ $\sigma x+2(2)$ \\ 5}

$\boldsymbol{\mu}$ the average of $\mathrm{x}, \boldsymbol{\mu y}$ the average of the variance of $\mathrm{y}$; $\boldsymbol{\sigma} 2 \boldsymbol{x}$ is the variance of $\boldsymbol{x}, \boldsymbol{\sigma} 2 \mathrm{y}$ the covariance of $\mathrm{y}$ and , $\mathrm{M} \times \mathrm{N}$ is the size of processed image. parameters are concerned but the visual quality is retained by Lee and Weiner filter which can be proved by the values of SSIM. Table No 1 shows that at medium noise density Arithmetic and mean filter shows good performance along with Weiner and Fuzzy filter in terms of statistical as well as visual Parameters. The performance of various spatial enhancement approaches are analyzed and discussed. The experiments are carried out for calculation of parameters.

\section{PERFORMANCE ANALYSIS}

Performance analysis can be done based on following parameters. 1. Cause of Speckle noise 2. Based on Statistical parameters and Visual Parameters 3. The density of noise contamination Cause of Speckle noise is introduced at all stages of image processing. One of the main reason of speckle is poor contact of sensor and body. In such types of noise the adaptive filters are best as they accommodate changes in local properties as well as the nature of the sensor. The center weighted adaptive median filter filter shows better performance as far as adaptive filters are concerned but the image is highly degraded during the reconstruction. The visual quality of an image is poorer at high noise density though statistical values are consistent. Different noise densities such as low Noise Density (LND) Medium Noise Density (MND) and High Noise Density (HND). This classification is based on level of contamination introduced in an image. Table No 2 also shows that Lee and Weiner filter performs well and consistently. Statistical and Visual Parameters Performance analysis is done based on statistical as well as visual parameters. Parameters like PSNR, MSE,RT describes the performance of all mentioned filters whereas UQI,SSIM describes performance in terms of visual quality. Lee filter and Weiner filter gives overall consistent performance. But at all the noise densities Weiner filter gives best statistical values as well as retains visual quality of an image. The density of noise contamination The performance can be evaluated based on level of contamination. Since speckle noise is multiplicative noise it degrades the image up to high extent. As level of contamination increases, the performance of median and mean filters decreases in terms of image statistics as well as visual quality. At low noise density Lee filter shows the best performance. Arithmetic mean filter, Weiner filter and fuzzy filters also shows comparatively good results. The PSNR values are comparatively for all existing filters ranging from 22to $26 \mathrm{~dB}$ so at all noise densities Lee filter is the best as far as statistical center weighted median filter (ACWMF),Vector median filter(VMF), Arithmetic mean filter(AMF), Harmonic mean filter( HMF),Average mean filter(AVMF),Weiner Filter(WF),Fuzzy Filter (FF), Hybrid Filter (HF),Lee filter(LF)[11]. Table No 1 and Table No 2 represents summary of medium and high noise densities .The graphical analysis is given for easy understanding. The filters used for implementation are simple median filter(SMF),AdaptiveMedian Filter (AMF),Adaptive The computational result showed that Lee and Weiner filters have the best results Due to its nonlinear nature and adaptive property. It proves to be excellent both speckle reduction and detail preserving properties. The response of the filter varies locally with the coefficient of variation. In case of low coefficient of variation, the filter is more average like, and in cases of high coefficient of variation, the filter attempts to 
preserve sharp features by not averaging. Mean , Median and reasonable result as these filters do not take all the statistical characteristics of the image into consideration. The advantage of the median filter is its simplicity and algorithmic straight forwardness.

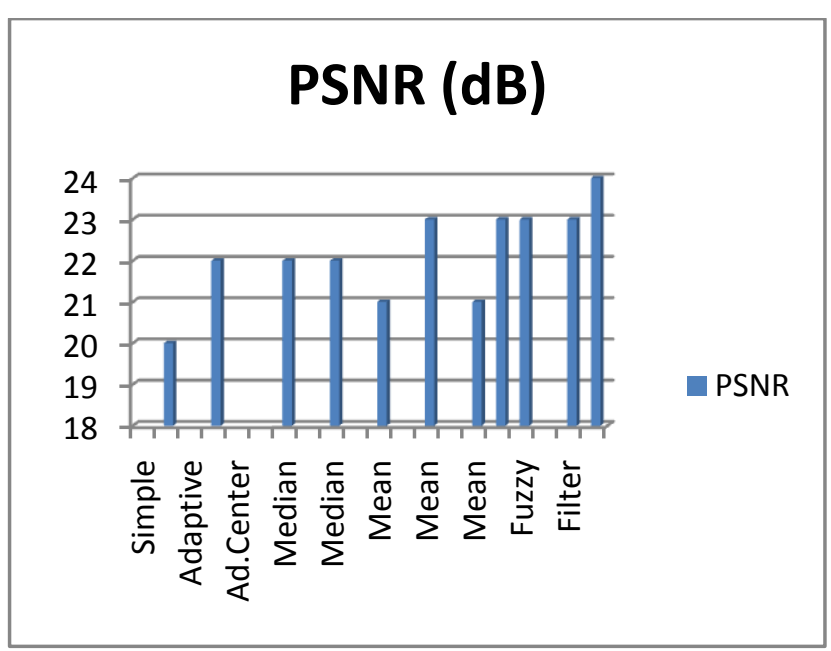

Figure No.1

\begin{tabular}{|c|c|c|c|c|c|c|c|}
\hline \multicolumn{8}{|c|}{ For Medium Noise Density $(0.4 \%)$} \\
\hline \multirow{2}{*}{ Sr.No } & \multirow{2}{*}{$\begin{array}{c}\text { Filtering } \\
\text { Technique }\end{array}$} & \multicolumn{6}{|c|}{ Image parameters } \\
\hline & & PSNR & MSE & RMSE & UQI & SSIM & RT \\
\hline 1 & $\begin{array}{l}\text { Simple } \\
\text { Median }\end{array}$ & 20 & 590 & 24 & 0.3 & 0.72 & 0.99 \\
\hline 2 & $\begin{array}{l}\text { Adaptive } \\
\text { Median }\end{array}$ & 22 & 460 & 21 & 0.5 & 0.61 & 0.99 \\
\hline 3 & $\begin{array}{l}\text { Ad.Center } \\
\text { Wt } \\
\text { Median }\end{array}$ & 22 & 452 & 21 & 0.5 & 0.76 & 0.98 \\
\hline 4 & $\begin{array}{l}\text { Vector } \\
\text { Median }\end{array}$ & 22 & 451 & 21 & 0.51 & 0.76 & 0.99 \\
\hline 5 & $\begin{array}{l}\text { Simple } \\
\text { Mean }\end{array}$ & 21 & 513 & 23 & 0.38 & 0.77 & 0.99 \\
\hline 6 & $\begin{array}{l}\text { Arithmatic } \\
\text { Mean }\end{array}$ & 23 & 294 & 17 & 0.37 & 0.77 & 0.99 \\
\hline 8 & $\begin{array}{l}\text { Avarage } \\
\text { Mean }\end{array}$ & 21 & 508 & 23 & 0.38 & 0.77 & 0.99 \\
\hline 9 & Wenier & 23 & 353 & 19 & 0.41 & 0.79 & 0.98 \\
\hline 10 & Fuzzy & 23 & 348 & 19 & 0.38 & 0.76 & 0.99 \\
\hline 11 & $\begin{array}{l}\text { Hybrid } \\
\text { Filter }\end{array}$ & 23 & 340 & 18 & 0.38 & 0.76 & 0.99 \\
\hline 12 & Lee & 24 & 265 & 16 & 0.42 & 0.79 & 0.99 \\
\hline
\end{tabular}

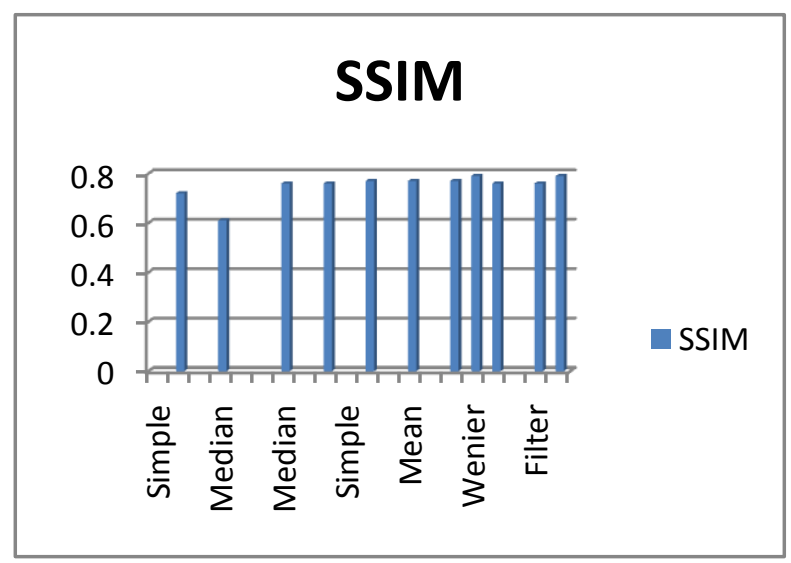

Figure No.2

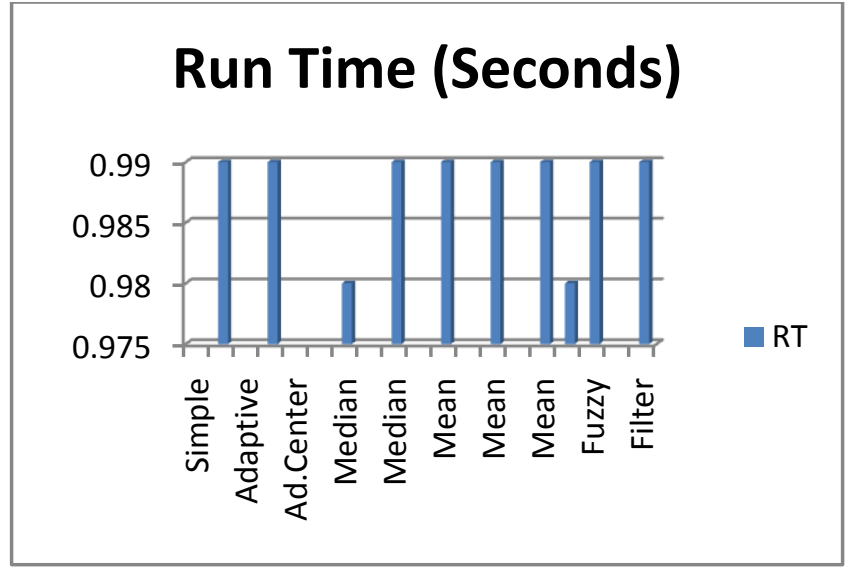

Figure No.3

\section{CONCLUSION}

The performance of noise removing algorithms is measured using quantitative performance measures such as MSE, SNR as well as in term of visual quality of the images. Many of the methods fail to remove speckle noise present in the medical image, since the information about the variance of the noise may not be identified by the methods. Performance of all algorithms is tested with medical images. The computational result showed that Weiner filter shows best computational as well as visual quality is retained as all the noise densities.

\section{REFERENCES}

[1] R.C. Gonzalez and R.E. Woods, Digital Image Processing. 2nd ed. Englewood Cliffs, NJ: Prentice-Hall; 2002

[2] A.K. Jain, Fundamentals of Digital Image Processing. Englewood Cliffs, NJ: Prentice-Hall; 1989

[3] Dr. R.K.Kulkarni PhD. Thesis Novel Restoration Techniques for Images Corrupted with High Density Impulsive Noise, NIT Rourkela

[4] C.P. Loizou and C.S. Pattichis "Introduction to Ultrasound Imaging and Speckle Noise," in Despeckle Filtering Algorithms and Software for Ultrasound Imaging, Morgan \& Claypool Publishers, 2008, pp. 3-23

[5] T.Ratha Jeyalakshmi and K.Ramar 'A Modified Method for Speckle Noise Removal in Ultrasound Medical Images' International Journal of Computer and Electrical Engineering, Vol. 2, No. 1, February, 2010 1793-8163

[6] Ahmed H. Samak 'A New Nonlinear Anisotropic Wiener Method for Speckle Noise Reduction in Optical Coherence Tomography' International Journal of Computer Applications (0975 - 8887) Volume 65No.12, March 2013

[7] Navjot Kaur1, Sunil Khullar 'An Approach for Speckle Noise Reduction from US Images using Enhanced Sticks Filtering' International Journal of Computer Science and Communication Engineering Volume 2 Issue 1 February 2013

[8] Chanchal Srivastava, Saurabh Kumar Mishra, Pallavi Asthana 'Performance Comparison of Various Filters and Wavelet Transform for Image De-Noising' IOSR Journal of Computer Engineering Volume 10, Issue 1 (Mar. - Apr. 2013), PP 55-63 
[9] Naman Chopra Mr. Anshul Anand 'Despeckling of Images Using Wiener Filter in Dual Wavelet Transform Domain' International Journal of Computer Science and Information Technologies, Vol. 5 (3) , 2014, 4069-4071

[10] P.S. Hiremath, Prema T. Akkasaligar 'Speckle Noise Reduction in Medical Ultrasound Images' http://dx.doi.org/10.5772/56519
[11] Mihir N.Dalwadi , Prof. D.N.Khandhar, Prof. Kinita H.Wandra 'Automatic Boundary Detection and Generation of Region of Interest for Focal Liver Lesion Ultrasound Image Using Texture Analysis' International Journal of Advanced Research in Computer Engineering \& Technology (IJARCET) Volume 2, Issue 7, July 2013 